\title{
The modeling of college students' psychological health based on statistical analysis
}

\author{
Huiling Wang \\ HaiNan College Of Software Technology, QiongHai, HanNan, 571400 China
}

Keywords: college students; psychological monitoring; abnormal behavior

\begin{abstract}
In order to further characterize the conditions of the college students' psychological health, this paper proposes the college students' psychological health models based on the statistical analysis techniques, which begin with the psychological issues triggered by the trivial events in the everyday life, consider the individual psychological abnormalities as the driving forces and analyze the impacts of the events relevance on students' psychological issues. The experimental results demonstrate the performance and impacts of the statistical analysis model for college students' individual psychological formation.
\end{abstract}

\section{Introduction}

The abnormal behaviors of the college students caused by the psychological issues are common in the college students' daily life. Usually, the trivial events in the daily life may impact the students' psychological conditions and cause psychological issues, such as the pressure in the college studies and occasional frictions. The students' psychological issues will not only impact the normal orders in the campus, but also will easily lead sudden mass incidents. The sociological studies ${ }^{[1 \sim 3]}$ have found the abnormal behaviors of the students are inevitable to trigger the psychological issues. It is important to figure out the reasons for psychological issues, carry researches on the prevention of the issues and mitigation measures to prevent accidental incidents from transferring to mass incidents, even worse, the violent incidents ${ }^{[4]}$.

This paper mainly focuses on the formative stage of the students' psychological issues. Based on the analysis of the reasons for the students' daily psychological issues, the individual abnormal activities are used to emulate the formation of the college students' psychological issues. The main objective of this paper is to predict the psychological issues and provide some theoretical basis for the relevant administrative departments.

\section{The model of mental issues formation}

\subsection{The familiar degree with mental issues}

The understanding of individual issues among students usually comes from the information exchange. The psychologists believe that the student population is the unit with mutual engagement. The whole student group can be divided into several small groups in which the individuals can exchange information. The individuals who do not understand the psychological problems will be impacted by the ones in the dominant positions.

Suppose the individual $i$ in the small group and it is used as the center of a circle. The individuals in the center area will be considered in the small group related to the individual $i$, which is the small group of $i$. In the group, the members in the dominant positions are considered as the members which can present the comprehensively explanation of the psychological issues. At time $t$, the members $\mathrm{CP}(\mathrm{i}, \mathrm{t})$ in the dominant positions within the small group of $\mathrm{i}$ can be expressed by

$$
C P(i, t)=\operatorname{argmax}((K E(j, t) * P(j) \mid j \in G(i)
$$

In the equation, $K E(j, t) \in[0,1]$ denotes the understanding degrees of the student $j$ on the psychological issues in the small group. If it equals 0 , it means the student knows nothing about the issues; if it's 1 , the student completely knows the psychological issues. $P(j)$ represents the willing of the individual to communicate with others. If $P(j)$ equals 0 , the individual $j$ does not communicate 
with others, otherwise, the student is very willing to communicate with others.

In the communication process, the students will gradually understand the psychological issues. If the members in the dominant positions know much more than the individual $i$, that is $K E(j, t)>K E(i, t)$. The understanding degrees of the individual $i$ at time $t+1$ on psychological issues can be defined as

$K E(i+1, t)=K E(i, t)+\operatorname{acp}(i) * \lambda * K E(j, t) * p(j)$

In the equation, $a c p(i)$ represents the acceptance and understanding degrees of the individual $i$ on the contents from the dominant members. There are duplicated contents between the individual $i$ and $j$, the randomly generated parameter $\lambda$ denotes the repeatability of the information.

\subsection{The mental issues triggered by events}

The psychological studies have found that the human beings are born with the abnormal psychology on model events and model information. The abnormal perception is the main driving force for individual sensing the trivial changes in the environment to quickly response, understanding the novel information and extracting information from the novel factors. The researches on the students' psychological issues formation found depression is the main psychology of individual psychological issues, which is the pre-event psychological experience of individuals involving in the events. In the researches of psychological issues escalation, the main reasons for the scale expansion mechanisms are the joined bystanders and the main psychology of them are curiosity, induction, resentment and bigotry. In the induced events, the spatial, time and event contents of the individuals have crucial impacts. In the perspective of event contents, the more crucial question of the individual, the more easily to induce psychological problems of the individual. The sudden accidents are the main fuses to form psychological issues.

Based on the previous analysis, this paper defines the depression as the main factor of psychological issues. For the quantification of the depression, the main impact factors are event contents, distances between the individuals and the events positions, and the understanding degrees of the individuals on events. The depression intensity $I_{\mathrm{S}}(I, t)$ of the individual $i$ at time $\mathrm{t}$ for not completely understanding the event $(K E(t)<1.0)$ is defined as the equation (3).

$$
I_{S}(t)=\left\{\begin{array}{cc}
1 & \text { dis }_{i, \text { eve }}<E_{V} \\
0 & \text { dis }_{i, \text { eve }}>2 * E_{V} \\
\frac{\gamma^{*} E C R^{b}}{\left(\text { dis }_{i, \text { eve }}-E_{v}+1\right)^{2}} & \text { other }
\end{array}\right.
$$

In the equation, ECR is the event content correlation, $E_{\mathrm{v}}$ is the distance for the individual sensing the events, namely the momentum caused by the main personnel of the events. $E_{\mathrm{v}}$ is defined as $E_{\mathrm{v}}=$ $\beta *$ agent-number ${ }^{1 / 2}$ in which agent-number is the number of personnel when the system is initialized. $D i s_{\mathrm{i}, \text { eve }}$ is the distance between the individuals and the events positions. $\mathrm{b} \in[0,1]$ is the emotional adjustment factor to reflect the emotional intensity. $\gamma \in[0,1]$ is the personalized adjustment factor. Based on the event correlation, $\gamma=$ random (ECR) can be randomly generated to reflect the depression degrees of different individuals on the events. The definitions of the depression intensity denote only the individuals in some area can sense the events and trigger the depression. The closer of the individuals to the events, the stronger the individual's psychological depression is.

\subsection{The selection of the evaluation parameters in the model}

This paper selects the Gaussian function as the core function and the corresponding Gaussian core function is expressed by

$$
K\left(\boldsymbol{X}_{i}, \boldsymbol{X}_{\boldsymbol{j}}\right)=\exp \left(-\gamma\left\|\boldsymbol{X}_{\boldsymbol{i}}-\boldsymbol{X}_{\boldsymbol{j}}\right\|^{2}\right)
$$

In the equation, $\gamma$ is the width parameter of the Gaussian core function.

SVM is used to model the college students' psychological issues. In order to obtain better performance, the parameters in the algorithm should be properly selected. The 3 parameters in the corresponding SVM model are 
(1) The regularized parameter $C$. This parameter directly determines the complexity of the model and the weights of the training errors in the objective function.

(2) The width parameter $\gamma$. This parameter indicates the non-linear projective degree from low-dimensional space to the high-dimensional feature space, which determines the structure of high-dimensional feature space.

(3) The non-sensitive loss factor $\varepsilon$. The smaller the parameter, the more number of support vectors $\mathrm{c}$, the more intense expression of solutions will be, vice versa.

There are a variety of selection methods for these three parameters. Many researchers introduced a number of advanced intelligent algorithms to automatically obtain these three parameters. In this paper, the cross-validation method is applied to determine these three parameters.

\section{Experiments and results analysis}

The contents triggering the psychological issues are various. The individuals have different attention and depression degrees. Normally, the bigger of the correlation between the events and the individual, the bigger of the probability of the individuals having psychological issues, the much more serious the psychological issues will be. The correlation degrees of the event contents are set from 0 to 1 . There are 120 students selected to evaluate the impacts of the events on the individuals. The experimental results illustrate when $\mathrm{ECR}=0$, the individuals will have psychological issues due to depression. With the time passed, the number of population with psychological issues has no obvious increment.

When the event contents and the psychological issues have some relevance, the individuals hope to control the process of the events. With the time of change, some psychological phenomenon will happen. With the increase of the relevance between the events and the individuals, the time of psychological issues will also increase. When the relevance degree of the event contents and the individuals reaches 1 , the serious psychological issues will happen.

In order to intuitively understand and analyze the impact of different events on the individual psychology, this paper selects the event contents relevance $E C R$ as $0.0,0.3,0.5,0.7$ and 1.0 respectively. The relationship between the number of individuals with psychological issues and the event contents relevance after 2000 unites of time is observed and shown in Fig. 1, which is basically the same as that in the real life. In the conditions with clear event conditions, the relevance of the events and the individuals is depended on the time of the psychological issues and severity. The closer of the events with the individuals, the more serious the psychological problems will arise.

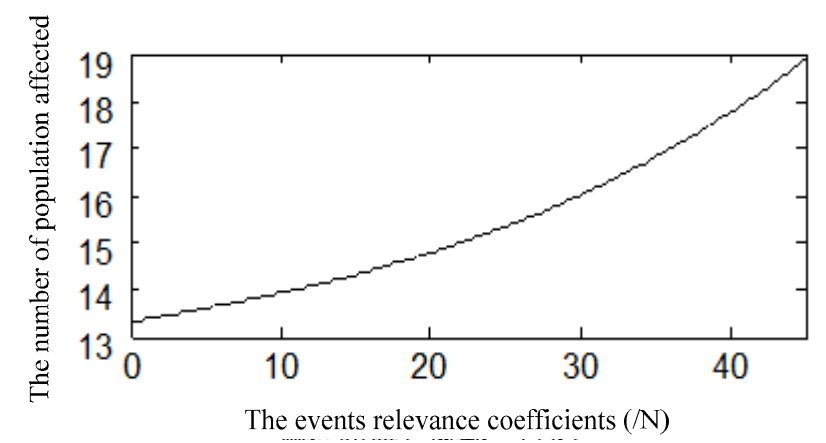

Fig. 1 Events correlation and aggregation number

\section{Conclusions}

This paper proposes the college students' psychological health models based on the statistical analysis techniques, which begin with the psychological issues triggered by the trivial events in the everyday life, consider the individual psychological abnormalities as the driving forces and analyze the impacts of the events relevance on students' psychological issues. The experimental results demonstrate the performance and impacts of the statistical analysis model for college students' individual psychological formation. 


\section{References}

[1] Xu Yao ya-li liu. Escalation of mass incidents and restrain mechanism study [J]. Journal of China national school of administration, 2011,01:17-21

[2] Chang Qin.the mass incidents dynamics model based on Agent research [J]. Journal of the Chinese people's public security university (natural science edition), 2010, 83:83-86.Epstein J M. Modeling civil violence: An agent-based computational approach [J]. Proceedings of the National Academy of Sciences of the United States of America, 2002, 99(Suppl 3): 7243-7250.

[3] Granovetter M. Threshold models of collective behavior[J]. American journal of sociology, 1978: 1420-1443.

[4] Wander Jager. Clustering and Fighting in Two-Party Crowds: Simulating the approach-Avoidance Conflict[J]. Journal of Artificial Societies and Social Simulation, 2001,4(3):1-18 